\title{
Clines Arc through Multivariate Morphospace
}

\author{
Brian K. Lohman, ${ }^{1, \star}$ Daniel Berner, ${ }^{2}$ and Daniel I. Bolnick ${ }^{1}$ \\ 1. Department of Ecology, Evolution, and Behavior, University of Texas, Austin, Texas 78712; 2. Zoological Institute, University of Basel, \\ CH-4051 Basel, Switzerland
}

Submitted June 23, 2016; Accepted November 10, 2016; Electronically published February 2, 2017

Online enhancements: appendix, video, Excel file. Dryad data: http://dx.doi.org/10.5061/dryad.25h9d.

\begin{abstract}
AвSTRACT: Evolutionary biologists typically represent clines as spatial gradients in a univariate character (or a principal-component axis) whose mean changes as a function of location along a transect spanning an environmental gradient or ecotone. This univariate approach may obscure the multivariate nature of phenotypic evolution across a landscape. Clines might instead be plotted as a series of vectors in multidimensional morphospace, connecting sequential geographic sites. We present a model showing that clines may trace nonlinear paths that arc through morphospace rather than elongating along a single major trajectory. Arcing clines arise because different characters diverge at different rates or locations along a geographic transect. We empirically confirm that some clines arc through morphospace, using morphological data from threespine stickleback sampled along eight independent transects from lakes down their respective outlet streams. In all eight clines, successive vectors of lake-stream divergence fluctuate in direction and magnitude in trait space, rather than pointing along a single phenotypic axis. Most clines exhibit surprisingly irregular directions of divergence as one moves downstream, although a few clines exhibit more directional arcs through morphospace. Our results highlight the multivariate complexity of clines that cannot be captured with the traditional graphical framework. We discuss hypotheses regarding the causes, and implications, of such arcing multivariate clines.
\end{abstract}

Keywords: migration-selection balance, morphospace, multivariate evolution, quantitative traits, threespine stickleback.

\section{Introduction}

The term "cline" is used to describe a gradual change in phenotype or genotype across a spatial transect (Endler 1977). For example, in Australian populations of Drosophila melanogaster, temperature tolerance and chromosomalinversion frequencies change steadily across a continentwide north-south axis (Hoffmann et al. 2002). At a smaller scale, the shell shape of Littorina saxatilis snails varies across a few-meter vertical gradient in rocky intertidal habitats (Grahame et al. 2006). Across such disparate spatial scales, clines arise from a tension between homogenizing and divergent evolutionary forces. Genetic drift or selec-

* Corresponding author; e-mail: lohman@utexas.edu.

Am. Nat. 2017. Vol. 189, pp. 354-367. (C) 2017 by The University of Chicago. 0003-0147/2017/18904-57077\$15.00. All rights reserved.

DOI: $10.1086 / 690808$ tion may act to drive differences between populations. Gene flow, however, acts to homogenize adjoining populations (Slatkin 1985; Lenormand 2002). The net result of these opposing processes depends on their relative strengths but typically results in a gradual transition in allele frequency or phenotypic means across a geographic transect (Fitzpatrick 2013). Clines are therefore often used to gain insight into the relative strengths of various evolutionary forces, the origin of intraspecific genetic variation, and early stages of parapatric speciation (Endler 1977; Caisse and Antonovics 1978; Gilchrist et al. 2001; Hoffmann et al. 2002; Stinchcombe et al. 2004; Umina et al. 2005).

Most theory and empirical research on clines focuses on spatial variation in particular loci or traits (Endler 1977; Slatkin 1978; Barton and Hewitt 1989; Barton 1999; Barton and Keightley 2002). When studies do examine multitrait or multilocus divergence, they typically reduce such multidimensional data to a single axis, such as a principal-component (PC) axis, discriminant-function axis, or genome-wide $F_{\text {ST }}$. Adopting this univariate view, clines are usually thought of in terms of monotonic (often sigmoidal) transitions in trait means or allele frequencies along transects through a landscape (e.g., fig. 1A; Slatkin 1978; Campitelli and Stinchcombe 2013; Geroldinger and Bürger 2015). When using independent traits (such as PC axes, which are mathematically orthogonal), we would expect each PC axis cline to resemble a typical univariate cline, although the cline's slope and inflection point may vary among traits or axes. For instance, if one trait is subject to stronger divergent selection, then its cline may be steeper and more strongly anchored to the boundary between habitats.

Recently, there has been growing interest in the dynamics of clines in genome-wide variation (Gompert and Buerkle 2011; Fitzpatrick 2013; Adrion et al. 2015), polygenic traits (Slatkin 1978; Barton 1999; Geroldinger and Bürger 2015), or multiple correlated traits (Stock et al. 2014). This is an important advance because adaptive divergence across an ecotone will usually entail evolutionary differentiation for many distinct traits. For instance, adjoining lake and stream populations of threespine stickleback (Gasterosteus aculeatus) differ in traits related to immunity, foraging, locomotion, 
defense, neuroanatomy, and sensory abilities (Berner et al. 2008, 2009; Bolnick et al. 2015; Jiang et al. 2015). These traits may be correlated to varying degrees (Berner et al. 2010) and may experience unequal selection intensities, resulting in more complex forms of phenotypic change across a landscape.

The multivariate nature of adaptation means that clines should be plotted in multivariate morphospace that better reflects the spatially varying relationship among traits. For a data set composed of $N$ morphological traits measured along a cline, we can plot the multivariate trait mean ("centroid") for each sample location as a point in an $\mathrm{N}$-dimensional morphospace. A transect across a landscape can then be represented as a path through that morphospace: a series of headto-tail vectors connecting successive sampling locations (fig. $1 B$ ). One can then compare the lengths or angles between successive vectors in morphospace (Collyer and Adams 2007; Adams and Collyer 2009). This focuses our attention on the successive changes between each sequential sampling location. We call this a "successive-vector" representation of a cline.

Alternatively, one can plot vectors that all start at a single reference location (e.g., one habitat) but end at different sample locations (fig. 1D). This second approach, which we call a "cumulative-vector" cline, is useful because the vector lengths measure the cumulative magnitude of trait change for points along the cline. These vector lengths can themselves be plotted as a function of distance, without restricting the analysis to a single axis through multivariate space (fig. $1 F$ ). The angles between cumulative cline vectors measure the extent to which the orientation of divergence (weighting of various traits) shifts as one moves along the cline. Angles near 0 mean that each successive sample point diverges along the same combination of traits. In such instances, a single univariate axis can accurately represent the cline. We use the term "linear cline" to describe such clines that progress in a single consistent direction through morphospace, with increasing distance across the landscape.

Deviations from linear clines can be tested with a permutation-based statistical test (Collyer and Adams 2007) to evaluate the null hypothesis that cumulative vectors all point in the same direction (angle of 0 ). Significant variation in vector angles ("nonlinear clines") indicates that different combinations of traits are most divergent at different sample locations. Nonlinear clines can come in two forms. First, as one moves along a cline the vectors might fluctuate irregularly through morphospace, pointing in many different directions as one moves down the transect (a "wobbling cline"). Second, a series of cumulative vectors might systematically rotate in a particular direction through space (an "arcing cline"). These alternatives can be evaluated by plotting vector angle (relative to a consensus vector) as a function of transect location (fig. $1 E$ ). If the cumulative vectors point in different directions through morphospace, then a univariate axis such as a discriminant-function axis will overlook some appreciable fraction of the evolutionary divergence in the system. Indeed, further interpretation of the shape of evolutionary trajectories has been called for (Collyer and Adams 2013), especially with regard to possible mechanistic explanations for such observations in natural populations.

We wished to determine whether multivariate clines are linear, tending to follow a single, progressively longer trajectory through morphospace, or whether the clines are generally nonlinear. If clines trace nonlinear paths through morphospace, do these paths form directional arcs (arcing or rotating clines), or do they wobble irregularly around a major axis of divergence? We first present a quantitative genetic simulation of clinal divergence with migration and selection, showing that multivariate clines may form linear paths through morphospace. However, unequal selection on different traits or other asymmetric evolutionary forces can lead to nonlinear, arcing clines. We then tested for nonlinear clines by analyzing empirical, multivariate morphological data from parapatric lake and stream populations of threespine stickleback. In both our simulation and the reanalysis, we employed a recently developed statistical framework for detecting differences in vector orientation and magnitude in space (Adams and Collyer 2009; Collyer and Adams 2013), allowing us to distinguish linear, arcing, and wobbling clines.

\section{Model \\ Model Overview}

We use an individual-based quantitative genetic simulation to illustrate the potential for clines to trace nonlinear paths through multivariate space. We began by outlining a standard univariate model of migration-selection balance along a cline. We then expand this to a bivariate cline to show that, given certain assumptions, bivariate clines can generate a simple linear trajectory through morphospace. However, relaxing these assumptions causes clines to arc nonlinearly through bivariate morphospace. We present a bivariate model because it is the simplest multivariate morphospace to discuss and graph, but the concepts illustrated with this model readily extend to higher dimensional settings, such as the empirical system described below.

We modeled divergent selection acting on one or more quantitative traits in populations inhabiting two adjacent habitats. Model details are provided below. A large "continent" population in the first habitat exchanges migrants across an ecotone with a linear series of smaller populations (an "archipelago") in the alternate habitat. The model framework is inspired by the empirical system analyzed below (stickleback in a large lake that drains into a long outlet stream). As we discuss in "Discussion," however, the notion 


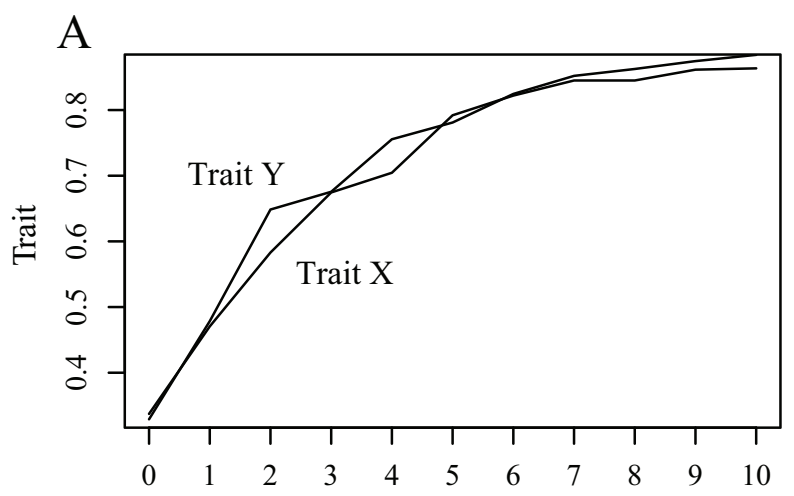

Distance
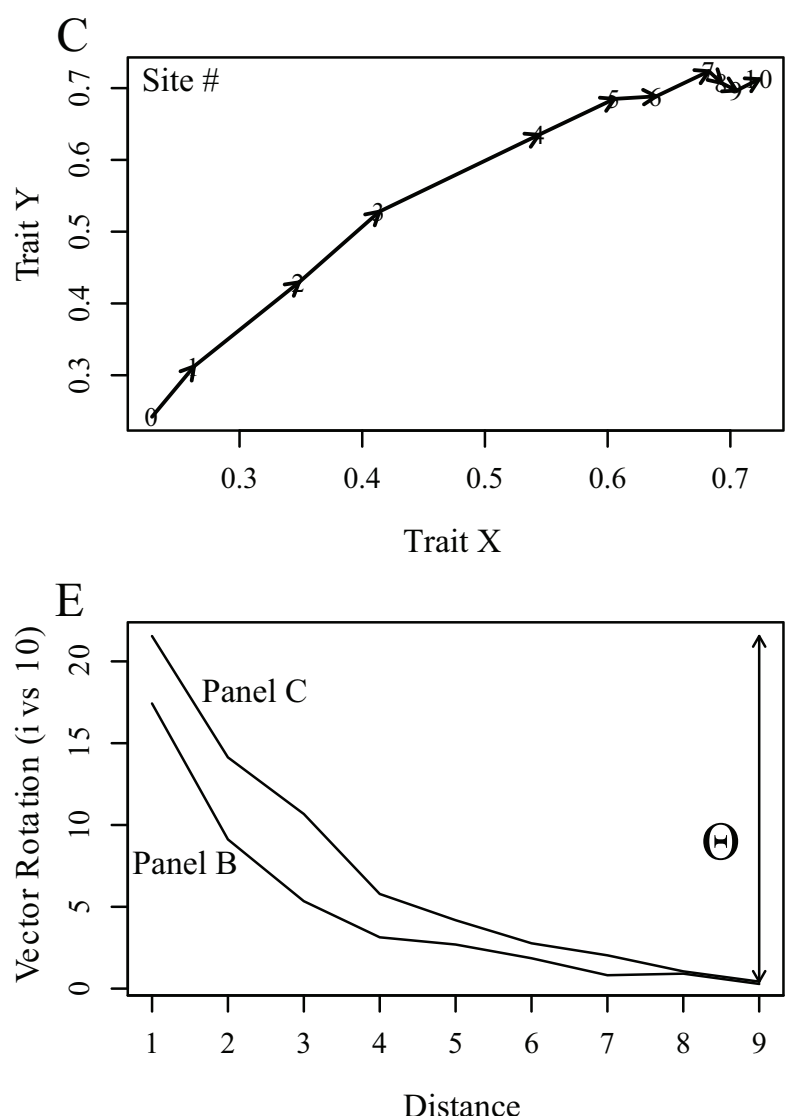

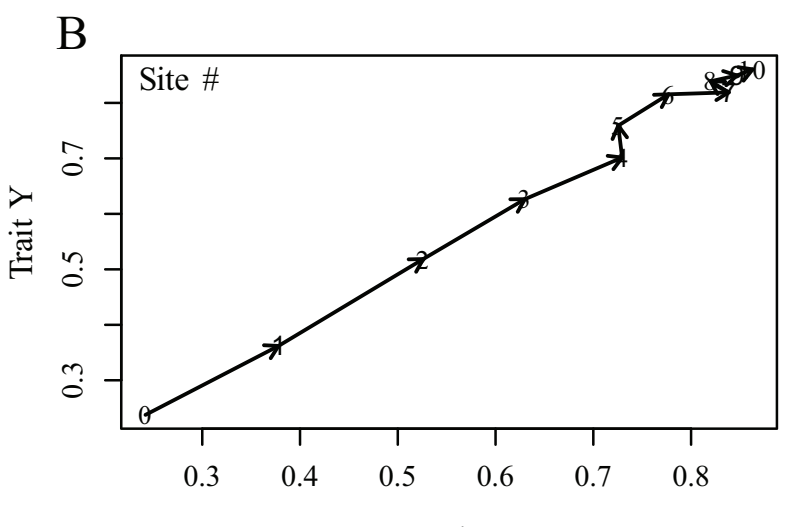

Trait X

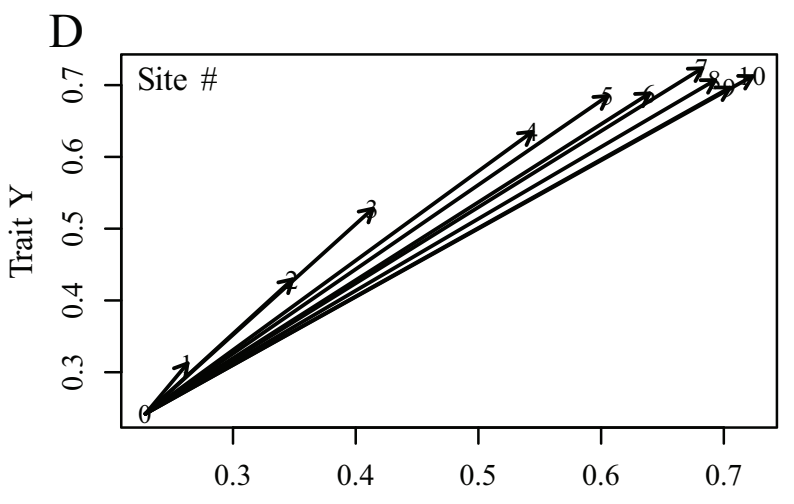

Trait X

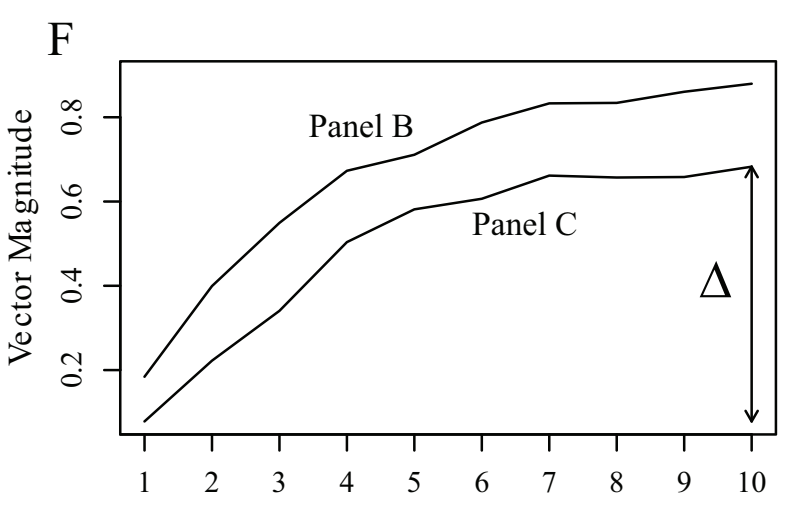

Distance

Figure 1: Example simulation outcome illustrating the multivariate behaviors of clinal divergence between a large "continent" population (site 0 ), and successively more distant populations along an archipelago (equivalently, a lake and stream). A, Two independent phenotypic traits, $\mathrm{X}$ and $\mathrm{Y}$, were assumed to be under divergent selection with gene flow, with phenotypic optima of 0 in the continent (site 0 ) and 1 in the stream (sites 1-10). When the traits are subject to equivalent selection and equal dominance, they both diverge at roughly identical rates along the cline (albeit with some weak stochasticity due to drift). $B$, The same data as in $A$ in bivariate morphospace, with vectors to connect each successive sample location. The vectors are long at first (representing a high marginal rate of divergence near the start of the cline) and shorter as the two traits approach their respective optima and gene flow from the continent is weak. However, because of the symmetric pressures experienced by traits $\mathrm{X}$ and $\mathrm{Y}$, all vectors point in roughly the same direction (stochasticity becoming relatively more effective at the most distant sites, where selection weakens as the population means approach their optima). This is a nonrotating cline. $C$, If we instead assume stronger selection on $\mathrm{Y}$ than on $\mathrm{X}$, the former trait approaches its optimum more quickly (initially greater change along the $\mathrm{Y}$ axis), and only farther down the archipelago, where immigration is reduced, does trait $\mathrm{X}$ evolution become relatively strong (as $\mathrm{Y}$ is already closer to the optimum). This is a rotating cline. $D$, The evolutionary trajectory shown in $C$ can also be plotted as a series of cumulative vectors connecting each archipelago location to the continent. This plotting strategy more clearly highlights the total magnitude of divergence along 
of nonlinear clines in morphospace should apply generally to a wider variety of spatial gradients.

\section{Analysis of Simulated and Empirical Clines}

To analyze the results of our simulations (and our empirical data), we analyze variation in the length and orientation of cumulative vectors, from the continent (lake) to successively more distant archipelago (stream) sites. Using cumulative vectors is particularly appropriate for continentisland models, for which the continent represents a clear baseline for comparison. We use two quantities to describe the behavior of the simulated and real clines. First, the length $(\delta)$ of each vector (e.g., from the continent to a given island) can be calculated with Euclidian distance in morphospace. Note that $\delta$ can be calculated for univariate, bivariate, or multivariate morphospace. Second, the dot product of two vectors can be used to measure the angle between any two vectors $\left(\theta_{i, j}\right)$. Note that vector angles are meaningful only for bi- or multivariate trait space. This angle requires a standardized reference vector against which all other continentisland vectors are compared. The reference might be the vector from the continent to the most distant archipelago site (our choice for this study), but in principle one might instead use a reference vector from the continent to the phenotypic centroid of the entire archipelago. Here, we opt to focus on calculating the angle $\theta_{i, 10}$ between an $i$ th cumulative vector (from the continent to the $i$ th archipelago site) and the vector from the continent to the most distant archipelago site $(i=10)$.

Using these angles, we can now quantitatively define different types of multivariate cline. First, a linear trajectory through morphospace occurs when all cumulative clines (or successive clines) have the same orientation in morphospace and as a result have angles $\theta_{i, 10}$ that are all indistinguishable from 0 . This linear trajectory serves as a null hypothesis that can be tested with permutation-based measures of significance for angles between vectors (Collyer and Adams 2007, 2013; Adams and Collyer 2009). Rejecting this null hypothesis for one or more sites along a cline implies that the cline forms a nonlinear trajectory through morphospace.

These nonlinear clines could arise in two ways. First, the cumulative vectors could progressively rotate in a consistent direction through morphospace as one moves along the archipelago. This rotation can be examined by plotting $\theta_{i, 10}$ as a function of cline location $i$. If a cline rotates through morphospace, then $\theta_{i, 10}$ will be large for small $i$ (sites closest to the continent), and as one moves farther away along the archipelago $\theta_{i, 10}$ should decline progressively toward 0 (as illustrated in fig. $1 E$ ). Clines that arc more strongly will tend to have a greater total rotation. We measure total rotation as the angle between the cumulative vectors from the continent to the first and last archipelago sites $\left(\theta_{1,10}\right.$, or simply $\left.\theta\right)$. Alternatively, nonlinear clines can "wobble," with vectors that fluctuate randomly around a reference trajectory. This second type of nonlinear cline is supported if one finds that vector angles $\theta_{i, 10}$ for successive sites do not exhibit a consistent decreasing trend with clinal location $i$.

We can also plot the vector length $\delta$ as a function of transect location (fig. $1 F$ ). For both univariate and multivariate clines, the typical expectation is that $\delta$ is initially small at the first cline sites because of the constraining effect of gene flow. The value of $\delta$ increases with greater distance from the continent (e.g., declining effect of gene flow from the other habitat) and should asymptotically approach a maximum as one moves far from the continent, because gene flow becomes very weak, allowing residents to approach their adaptive trait optima. For a summary statistic, we define $\Delta_{i, j}$ to be the difference in magnitude between any two vectors for archipelago sites $i$ and $j$. This should be large when the first and last sites along the archipelago are compared $\left(\Delta_{1,10}\right.$, hereafter $\left.\Delta\right)$ and should decrease toward 0 as one moves farther from the continent along the archipelago (fig. $1 F$ ). A large $\Delta$ implies a strong constraint by gene flow close to the continent but increasing trait divergence as one moves along the cline. A small $\Delta$ indicates either that most phenotypic divergence occurs at the continent-archipelago boundary or that there is little divergence at all.

In principle, similar summary statistics could be calculated relative to a consensus vector composed of many sample locations rather than just the most distant one. A guiding principle should be ensuring precision (e.g., large sample size) when estimating the reference vector, because a large standard error in reference-vector estimation will influence all other metrics. However, the reference should comprise one or more archipelago sites that are minimally affected by gene flow from the continent, to maximize one's ability to infer phenotypic optima for the archipelago habitat. Conversely, populations subject to strong genetic drift may tend to exhibit stochastic variation in vector direction, and this

the cline and the rotation in cline direction as we proceed from the first site to the last. $E$, Another way to visualize the rotation of cumulative clines is to plot the angle $\Theta$ between each cumulative vector (from $D$ ) relative to a reference vector (here, the most distant archipelago sample from site 10). That is, we plot $\theta_{i, 10}$ as a function of location $i$. For rotating clines through morphospace, initial sites in the archipelago will have a large angle relative to the most distant continent-island vector, and this angle will decay toward 0 as one proceeds along the archipelago, as illustrated here. Here, we plot the data from clines in $B$ and $C$ (lower and upper curves, nonrotating and rotating, respectively). $F$, A second measure of cline change through morphospace is to calculate the length of each vector, as a function of cline location. The cumulative amount of trait divergence at the farthest sampling location is $\Delta$, which was shorter for the rotating cline. 
may be most severe farthest from the continent, where the effective population size is smallest.

\section{Model Details}

We used a stochastic, individual-based model consisting of a single large population in one habitat (e.g., a continent or lake) and a linear array of 10 smaller populations in a second habitat (e.g., an archipelago or stream). The continent population had 10 times as many individuals as each archipelago site. Each site was populated with diploid individuals, each represented as a vector of genotypes for 20 loci affecting trait $\mathrm{X}$ and 20 loci affecting trait Y. All 40 loci were independently assorting and of equal effect size (allele values of 0 or 1 ). Each quantitative-trait value was the sum of the additive effect of its 20 loci (40 alleles), rescaled to lie between 0 and 1. Below, we introduce the possibility of dominance, but we leave analysis of different genetic architectures (e.g., linkage, epistasis, variation in allelic effect sizes) for future investigation. All alleles were initially polymorphic (allele frequencies of 0.5 in both island and continent), and individuals were randomly generated within sites. Allele frequencies were tracked for each geographic location (the continent and 10 sites along the archipelago) through time, with random mating, migration, and selection.

We model the life cycle as follows. New individuals are born into each habitat patch, by drawing diploid genotypes at random from the patch's adult allele frequency, for each of the 40 loci. To maintain genetic variation we introduce a low rate of mutation that reverses the allele value from 1 to 0 or from 0 to 1 with a very small probability, $\mu$. We use nonoverlapping generations, so this new generation replaced the extant adults, at a prespecified carrying capacity (regardless of mean fitness). Next, these juveniles were allowed to disperse randomly. A fraction $m$ of individuals were randomly selected to disperse according to a steppingstone model (dispersal limited to adjacent sites). Half of these dispersers moved toward the continent, and half moved away. The ends of the transect were reflecting boundaries, so any migrants moving downstream from the last site were reflected back into their natal site. The migration rate is equal between all adjacent sites and involves a random sample of the available genotypes from a source population.

The newly formed populations (migrants and residents) are then subject to viability selection. Within each habitat, each individual's fitness (survival probability) depends on its distance from a habitat-specific local trait optimum:

$$
\omega_{i}=\frac{\left(X_{i}-\beta_{j}\right)^{2}}{\sqrt{\tau}},
$$

where $X_{i}$ is the individual's quantitative-trait value, $\beta_{j}$ is the phenotypic optimum in the local habitat, and $\tau$ is the strength of stabilizing selection about the phenotypic opti- mum ( $\tau$ is initially assumed to be equal in the two habitats; we relax this assumption in later simulations). Lake and stream optimum phenotypes are 0 and 1, respectively, because alleles are coded as 0 or 1 . All stream sites have the same optimum phenotype. We multiply the fitness values of the two traits to obtain a probability that each individual survived, and we use a random-number generator to choose survivors on the basis of their respective fitness values. Individuals who survive determine the end-of-generation allele frequencies within sites, which are used to produce the next generation as described above.

During each generation we tracked the mean phenotype in each site, but our analysis focused on quasi-equilibrium values after 1,000 generations. At the end of a simulation we calculated cumulative vectors through bivariate morphospace for traits $\mathrm{X}$ and $\mathrm{Y}$ and calculated the summary statistics mentioned above. We repeated these simulations for a variety of parameter values, considering varying rates of migration, dominance, and the effects of unequal selection on $\mathrm{X}$ and $\mathrm{Y}\left(\tau_{\mathrm{X}} \neq \tau_{\mathrm{Y}}\right)$. To examine the effect of any focal parameter, we held all other variables constant and ran 10 replicate simulations for each of several values of the focal parameter. For each simulation, we recorded both of our summary statistics, $\theta$ and $\Delta$, and reported the mean and standard error of 10 replicate simulations with a single parameter value. We then examined how $\theta$ and $\delta$ varied along the length of a given cline. To test for significant variation in vector orientation (nonlinear clines), we used permutation tests to evaluate the null hypothesis that $\theta$ is near 0 for all cumulative vectors along a cline. We then examined how summary statistics of cline shape $\theta$ and $\Delta$ vary across parameter values.

We considered variation in the rate of migration (for $m=0.001,0.01,0.05,0.1,0.15,0.2,0.25$, and 0.3 ) while holding $\tau=0.3$ in the continent and 0.1 in the archipelago sites, for both traits. The asymmetry in selection strength was arbitrary but reflected empirical results that divergent selection between disparate habitats may be asymmetrically strong (Hanson et al. 2016). We varied the strength of dominance, $d$, from 0 to 1 (in increments of 0.1 ), holding other parameters constant. For each locus, homozygotes for the 1 or 0 allele contribute 1 or 0 , respectively, to the final trait value, while heterozygotes have effect $d$. For convenience, we apply $d$ equally to all 20 loci. The continent-favored allele (0) is dominant over the archipelago-favored allele (1) at each locus when $d<0.5$, and vice versa when $d>0.5$. We expect that dominance should skew the value of the quantitative trait, and thus the overall shape of the cline, more quickly than otherwise expected. Finally, we varied the ratio of selection intensity on the two traits, $\mathrm{X}$ and $\mathrm{Y}$. Holding the strength of stabilizing selection on $\mathrm{Y}$ constant $\left(\tau_{\mathrm{Y}}=0.1\right.$ in both habitats), we varied $\tau_{\mathrm{X}}$ from 0.1 (strong, equal to that for $\mathrm{Y}$ ) to 0.6 (weak selection) in increments of 0.1 . We also considered the interaction between pairs of parameters, for 
example, factorially covarying dominance and the migration rate. Our simulation was constructed in $\mathrm{R}$, with code that is available in the Dryad Digital Repository, http:// dx.doi.org/10.5061/dryad.25h9d (Lohman et al. 2017).

\section{Model Results}

When migration is low and selection affects traits $\mathrm{X}$ and $\mathrm{Y}$ similarly, they evolve highly concordant clines (fig. 1A). As a result, for every additional change in $\mathrm{X}$ along the cline, there is a comparable change in Y. Regardless of whether these traits change by the same number of units in each step down the cline, this equal marginal effect means that the cline progresses along a single major axis in bivariate trait space (fig. $1 B$ ). There is, consequently, no appreciable rotation as a function of distance along the cline ( $\theta$ is indistinguishable from 0 throughout). However, because of increasing isolation by distance along the cline, the most distant locations are able to approach their bivariate phenotypic optima, whereas the nearest locations are constrained by gene flow from the nearby continent. Cumulative length, therefore, is shorter near the habitat boundary and longer toward the end of the cline. When the cline is viewed as univariate, this is seen as an asymptotic approach to the optimal phenotype with increasing distance along the cline. When it is viewed as a bivariate trajectory, we instead see a series of increasingly long vectors (approaching an asymptotic length) that all point in the same direction. Conversely, as the distant archipelago populations approach their phenotypic optima, their sequential vectors become progressively shorter. Thus, with symmetric selection and additive allelic effects, $d=0.5$, the bivariate cline can effectively be described by divergence along a single principalcomponent axis that behaves like a classical univariate cline.

In contrast, other parameter combinations yield arcing clines (fig. $1 C, 1 D$ ). For instance, when $\mathrm{Y}$ is subject to stronger stabilizing selection than $\mathrm{X}$ within each habitat, $\mathrm{Y}$ diverges more rapidly at first and more quickly approaches its local optimum. As a result, Y experiences relatively little change along the last few steps of the cline. In contrast, the constraining effect of migration is comparatively stronger for weakly selected trait X. As a result, the cline increases more rapidly along axis $\mathrm{Y}$ than along axis $\mathrm{X}$ for the first sites along the archipelago (where $\mathrm{X}$ is constrained by gene flow) and then more rapidly along axis $\mathrm{X}$ than along axis $\mathrm{Y}$ farther down the transect (where $\mathrm{Y}$ is already optimized). The morphospace cline therefore forms an arc, which can be seen both as a curve traced by sequential vectors (fig. $1 C$ ) and as increasing angles between the cumulative vectors (fig. 1D). This arcing behavior of the cline is reflected in the steady decrease in $\theta_{i, 10}$ as one moves along the cline (fig. $1 E$ ). The arcing clines illustrated in figures $1 C$ and $1 D$ produce a much steeper decrease in $\theta_{i, 10}$ along the cline. Thus, the metric $\theta$ is smaller for our arcing cline (fig. $1 C, 1 D$ ) than for the linear cline (fig. $1 A, 1 B$ ). Conversely, the vector length begins low and increases along the transect. This latter trend is comparable to a univariate cline, but in the rotating case the vector length may increase more rapidly along the cline and approach the asymptote more quickly (fig. $1 F$ ).

When migration is very weak, selection can dominate across the full length of the archipelago and gene flow causes very little deviation from the multivariate trait optimum for any of the sites along the archipelago. Therefore, the cline almost immediately points to its final target, and there is little elongation or rotation as one moves along the archipelago. At higher values of migration, however, gene flow introduces more continent alleles into the first island, pulling allele frequencies in the first island toward the continent optimum and introducing genetic variation among islands, thereby generating appreciable variation in length and orientation (fig. 2, top). Gene flow most strongly affects the archipelago sites nearest the continent, which exhibit correspondingly short vectors. Migration thus increases variation in vector length along the cline. The effect of migration on vector orientation is more complex, drawing on two related phenonema. First, gene flow from the continent into the archipelago introduces more maladaptive genetic variance into nearby sites than into distant sites. The resulting inflation of genetic variation at nearby archipelago sites means that sampling error is correspondingly greater. We are therefore likely to obtain a local sample average that deviates from the major axis of adaptive divergence. Second, estimates of vector angles are more sensitive to sampling error for short than for long vectors. For a given standard error in estimating $\mathrm{X}$ and $\mathrm{Y}$, sampling error will generate larger angular variation for a short vector. Thus, gene flow from the continent into the first archipelago sites both inflates the genetic variance (and thus sampling standard error) and shortens the vector to introduce more uncertainty in vector angle. This explains why migration generates greater angular variation between the first and last archipelago sites, even though it is not deterministically affecting one trait more than the other.

For conditions with moderate gene flow, this vector rotation is exacerbated by asymmetric selection for reasons described above (fig. 2, middle). When we varied dominance we saw little effect on vector rotation or chance in vector magnitude (fig. 2, bottom). We reserve, for future work, analysis of other factors that might dictate the shape of multivariate clines, including analyses of higher-dimensional morphospace, more complex genetic architectures, or other geographic arrangements.

\section{Empirical Analysis}

To test whether multivariate clines indeed arc through morphospace, we applied the analytical methods described above 

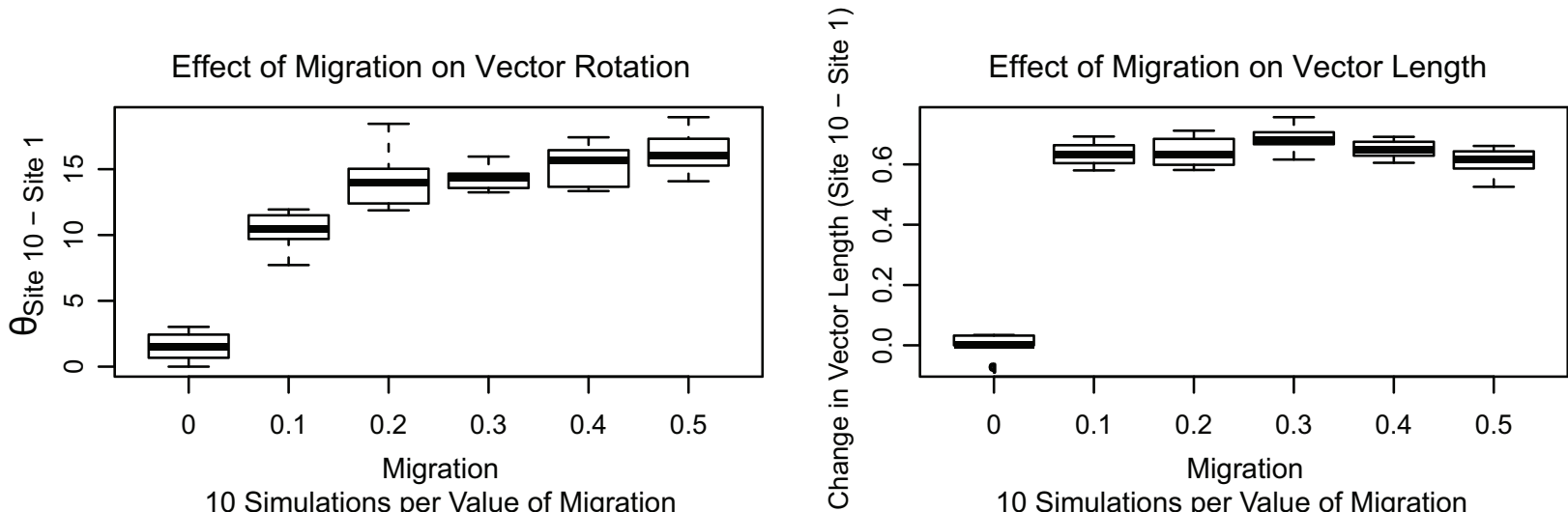

10 Simulations per Value of Migration

10 Simulations per Value of Migration

Effect of Asymmetric Selection on Vector Rotation
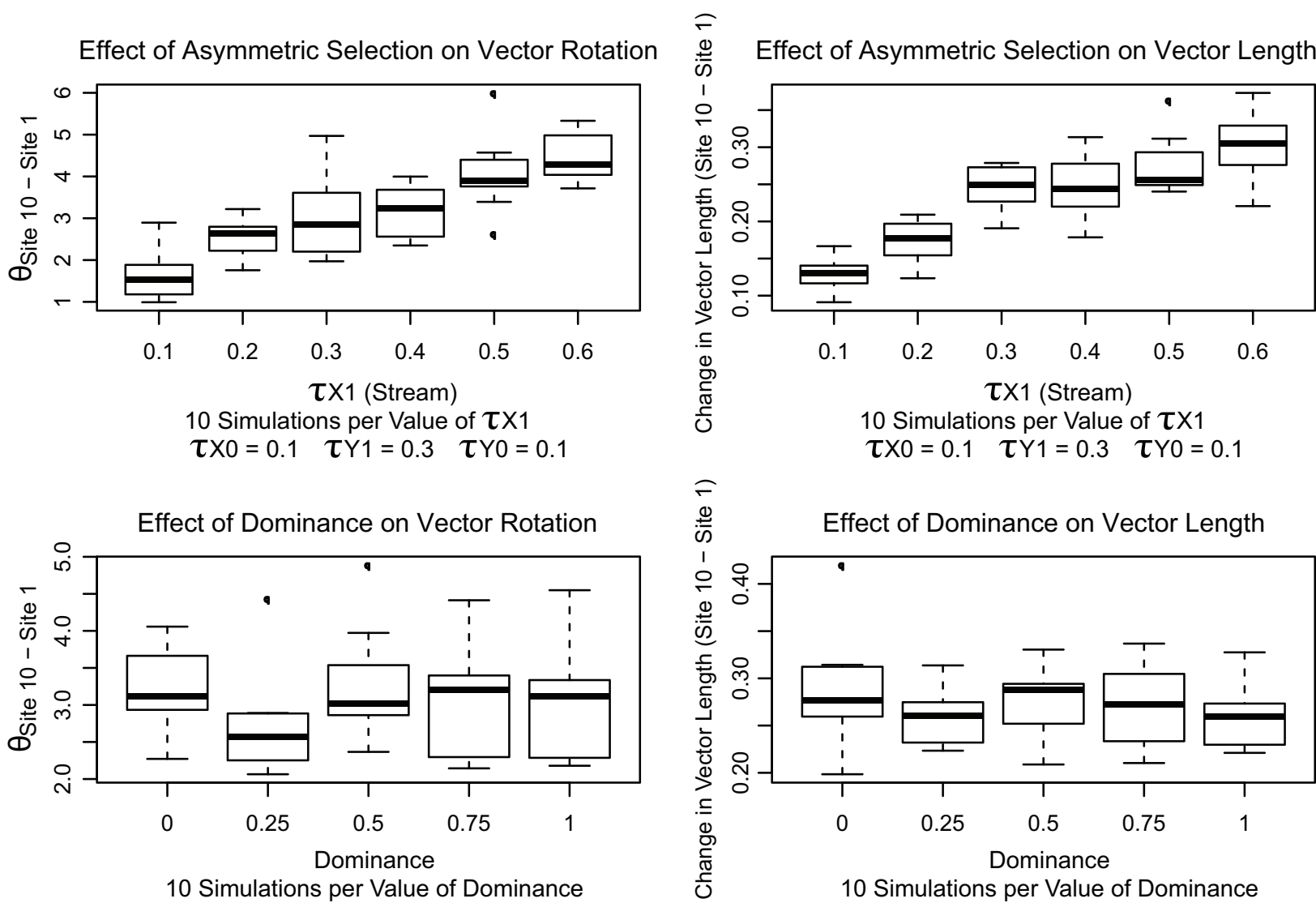

Figure 2: The effects of migration rate (top), asymmetric selection on traits $\mathrm{X}$ and $\mathrm{Y}$ (middle), and dominance (bottom) on the multivariate path of a continent-archipelago cline. Default values in simulations include: $N=100$ individuals in all sites, $\tau_{\mathrm{X} 0}, \tau_{\mathrm{Y} 0}=0.1, \tau_{\mathrm{X} 1}, \tau_{\mathrm{Y} 1}=0.3$, migration $=0.2, \beta_{\mathrm{X} 0}, \beta_{\mathrm{Y} 0}=0.1, \beta_{\mathrm{X} 1}, \beta_{\mathrm{Y} 1}=0.9$, and mutation rate $=0.0001$, and dominance for $\mathrm{X}$ and $\mathrm{Y}=0$. Note that smaller values of $\tau$ correspond to stronger stabilizing selection.

to existing clinal data from eight independent pairs of lake and stream populations of threespine stickleback. Parapatric lake and stream populations of stickleback make a good test case because they closely correspond to the continentarchipelago framework used in our simulations. Lake and stream populations are morphologically, genetically, and ecologically distinct (Lavin and McPhail 1993; Berner et al. 2008; Kaeuffer et al. 2012; Oke et al. 2016) and have been shown to experience divergent selection (Hendry et al. 2002). Lake stickleback are characterized by shallow, streamlined 
bodies and longer, more numerous gill rakers, for feeding on limnetic prey. Stream stickleback are deeper bodied and have fewer, shorter gill rakers, for feeding on benthic prey (Reimchen et al. 1985; Lavin and McPhail 1986, 1993; Thompson et al. 1997; Hendry et al. 2002; Hendry and Taylor 2004; Moore and Hendry 2005; Moore et al. 2007; Berner et al. 2008; Bolnick and Paull 2009). Both body shape and gill raker morphology are heritable traits, and differences in these traits between stickleback populations have a genetic component (Peichel et al. 2001; Albert et al. 2008), further supported by heritable differences between lab-reared lake and stream stickleback (Sharpe et al. 2008; Berner et al. 2011; Oke et al. 2016). Because adjoining lake and stream populations readily exchange migrants, individual morphological traits frequently exhibit classic univariate clines similar to those seen in figure $1 A$ (Moore and Hendry 2005; Berner et al. 2009; Bolnick et al. 2009). Here, we ask how these known phenotypic clines behave when plotted in multivariate morphospace. Specifically, do clines proceed linearly through morphospace, simply elongating as one gets farther from the continent (lake) and migration constraints get weaker (e.g., fig. $1 B$ )? Or do clines arc through morphospace, with different trait combinations driving lakestream divergence at different locations along the stream (e.g., fig. 1D)?

\section{Methods}

We reanalyzed data from a previously published study, Berner et al. (2009), using a modified version of the analytical method described in Adams and Collyer (2009): a statistical framework for analysis of vectors in space, estimating significance for differences in vector orientation and magnitude; in all cases the null hypothesis is that there is no difference in orientation or magnitude between vectors. The data from Berner et al. (2009) described morphological variation along eight independent lake-stream clines. Each lake drains into an outlet stream, and stickleback occupy both habitats. Morphological data were taken for 20 fish from each lake and from six sites along a transect down each stream. The mean distance between adjoining stream sample sites was $511 \mathrm{~m}, \mathrm{SD}=310 \mathrm{~m}$. The maximum distance between the lake and the farthest stream site varied by lake and was between 1,540 and 4,390 m, depending on the available length of outlet stream. Morphological data in our analysis included gill raker number, a heritable trait associated with benthic or limnetic feeding, as well as body shape scores (relative warps 1-5) from geometric morphometric analysis (see Berner et al. 2009 for full details). We followed Berner et al. in retaining only the first five relative warps, which capture the substantial majority of phenotypic variance within any given lake-stream pair. Includ- ing gill raker number as well, we have a six-dimensional morphospace.

We performed a principal-components analysis on the correlation matrix of phenotypic data (gill raker number and relative warps 1-5) separately for each of the eight lakestream pairs. For illustration purposes, we plot the clines in bivariate morphospace, using PC1 and PC2. However, our calculations of vector lengths and angles were in the six-dimensional morphospace allowed by these data (except where noted). We described changes in phenotype between the lake and each stream site as a vector whose attributes could be compared to those of other such vectors with the same Euclidian-distance and dot-product methods employed in our simulations. In addition, we used Adams and Collyer's (2009) permutation procedure (randomizing residuals) to test for statistically significant differences in vector length and orientation between all cumulative vectors along a given cline. Using these permutations, we tested the null hypothesis that the cumulative vectors all point in the same direction ( $\theta$ is indistinguishable from 0 throughout). We then tested whether the vector angle (relative to that of the most distant lake-stream vector) decreases as a function of location along the cline, as expected for a rotating cline. Alternatively, random fluctuations in $\theta$ along the cline's length imply "wobble" rather than rotation. All data analysis was carried out in R. We pooled both sexes for these analyses, following (Berner et al. 2009), who found comparable clines for both sexes.

\section{Results}

Plotting these well-studied clines in a multivariate morphospace provided a surprising insight. Classical theory predicts that clines should elongate along a single major axis, with minimal divergence in orientation and consistent changes in magnitude. However, successive sample sites downstream can be substantially different from one another, in terms of both divergence magnitude (as expected from migrationselection balance theory) and orientation. Some clines progress along a single major axis of lake-stream divergence, while others involve significantly different orientations in morphospace (fig. 3; also see video A1, available online). Along a given transect, cumulative vectors are significantly different from each other in orientation and/or length (table S1, available online).

Most dramatically, in the McCreight transect stream, locations 1, 3, 5, and 6 all had similarly higher values of PC2 (relative to the lake), whereas stream location 2 had lower values of PC2 and stream site 4 had vastly higher values of PC1 than all other sites (but did not differ from the lake along PC2). As a result, the vectors from the lake to sites 1, 3,5 , and 6 are all fairly similar, but the vector from the lake to site 2 is in the polar opposite direction, and the lake-site 4 vector is orthogonal to the others. However, because these 

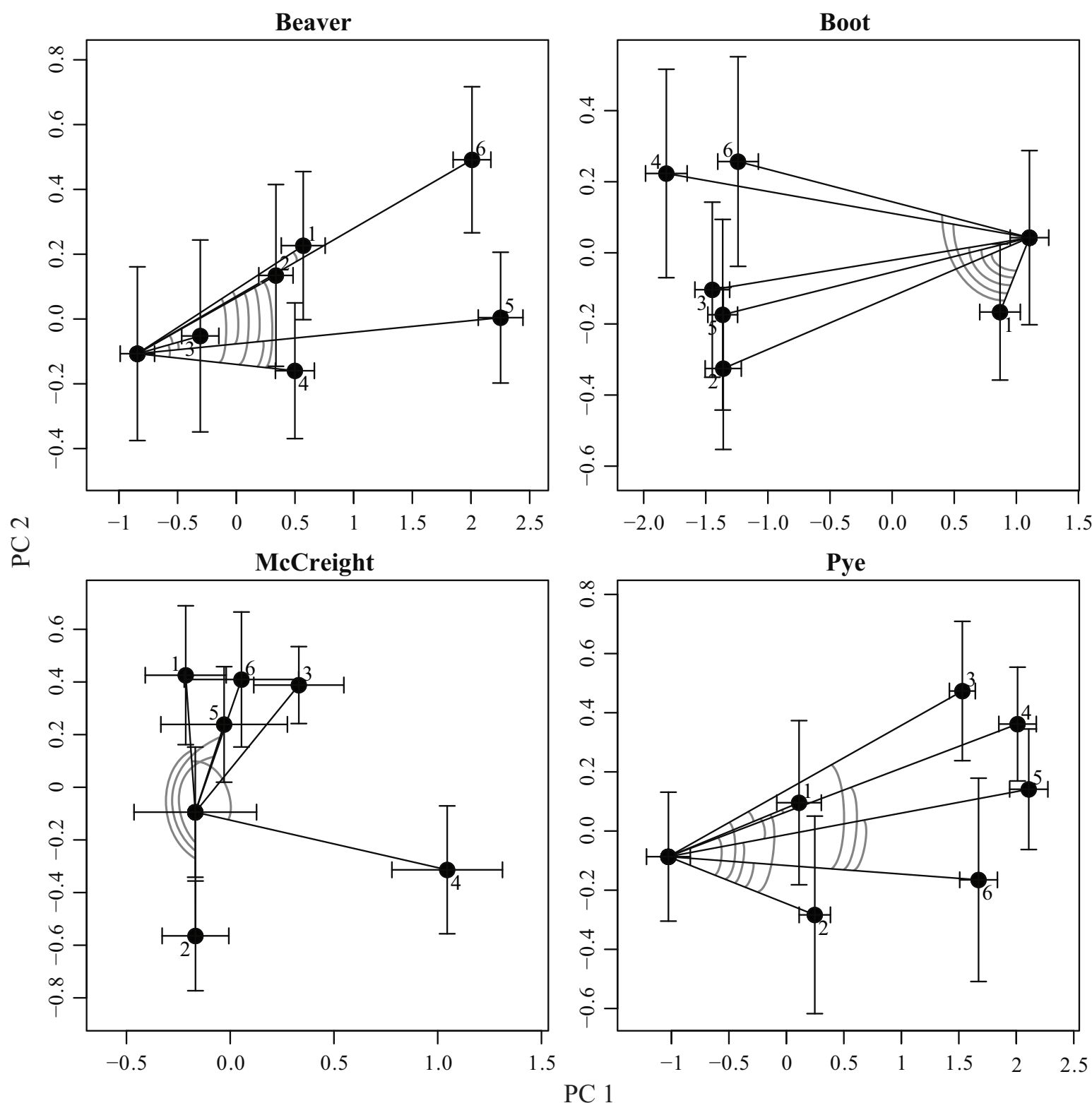

Figure 3: Four of the eight lake-stream clines of threespine stickleback, using data from Berner et al. (2009), represented as divergence in the first two principal-component axes (PC1 and PC2). A three-dimensional representation of one of these clines is in table S1, available online. All eight pairs are provided in figure A1, available online. The multivariate centroid for the lake population for each pair is plotted as an unnumbered circle, while the stream sites are plotted as numbered circles. Standard-error bars represent uncertainty in these centroid estimates. We plot the cumulative vectors from the lake to each successive stream site along the cline. Arcs identify pairs of vectors whose orientations are significantly different $(\theta>0)$ in the full multivariate morphospace (not merely the plotted bivariate space). The stream sites are numbered sequentially from 1 (closest to the lake) to 6 (farthest from the lake). See Berner et al. (2009) for details of sampling locations.

angles do not align cleanly along an order downstream, the lake-stream cline "wobbles" rather than tracing a smooth arc, as in our model (fig. 1D). Likewise, the Beaver lakestream pair exhibits a wobbling cline: the vectors from the lake to sites 1, 2, and 6 are all in the same directions (low $\theta \mathrm{s}$, but that to 6 is longer), and the vectors from the lake to sites $3-5$ point in mutually similar directions (that to 5 is the longest). However, these two clusters of vectors point in significantly different directions from each other, resulting in a wobbling cline through morphospace.

The Boot and Pye lake-stream pairs trace somewhat more steady arcs, showing significant differences in orien- 
tation but not all in the same direction. Boot stream, for instance, has smaller values along PC1 (relative to the lake) but variable directions along $\mathrm{PC} 2$, with the nearest sites having mostly smaller PC2 values and more distant sites higher PC2 values. Likewise, in Pye there is a fairly smooth arc as one moves from site 1 to site 6 (with site 2 generating some "wobble" in direction).

Our models suggest that when there is an arcing cline, this should be visible as a high-angle $\theta$ when comparing the first and last lake-stream vectors but that $\theta$ should rapidly drop to lower angles, approaching 0 , farther downstream. We observed this trend for Boot and, to a lesser degree, Pye. But, averaging across all eight lake-stream pairs, we observed no systematic decline in vector angle (relative to the most distant site) with distance along the transect (fig. 4, left). Thus, there is no shared tendency for lakestream pairs to arc through morphospace (at least for the traits and sites examined by Berner et al. 2009). On the other hand, there is a general (albeit noisy and not statistically significant) trend for vector lengths to increase with distance downstream (fig. 4, right). This averaged trend overlooks the fact that there is a strong asymptotic increase in vector magnitude in some pairs (even those that continue to rotate afterward, e.g., Pye), whereas in other pairs the lake and stream are about fully divergent immediately (e.g., McCreight) but continue to change orientation afterward.

\section{Discussion}

Although clines are most often represented as progressively greater divergence along a single trait axis, our simulations and data suggest that this is an oversimplification. Following a suggestion by Collyer and Adams (2013), we examine the trajectory of clines through multivariate morphospace.
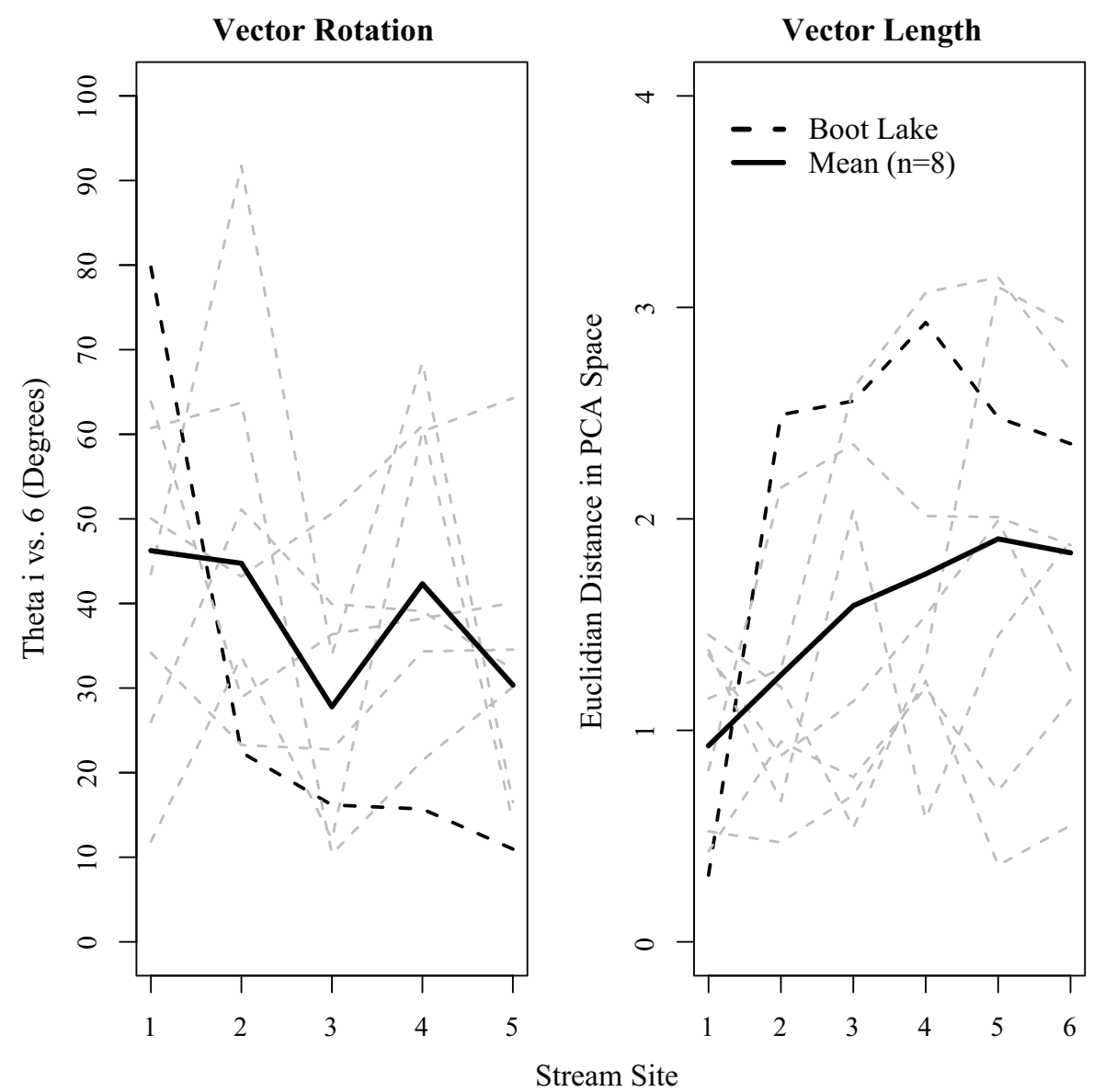

Figure 4: Left, there is a weak (nonsignificant) trend for $\theta_{i, 6}$ (relative to the most distant site) to decline with distance along the cline, as predicted by our model (fig. $1 E$ ). The trend for each lake-stream pair is plotted as a dashed line (highlighting Boot Lake, which exhibits the clearest rotation behavior in multivariate space (not just bivariate space plotted in fig. 3). The dark solid line represents the mean of the eight lake-stream pairs. Right, there is a significant trend for $\delta_{i, 6}$ to increase along each of the eight clines, consistent with our model, in which the constraining effect of gene most strongly affects the first sites in the archipelago and weakens, allowing more substantial divergence, farther down the cline even though the cline continues to rotate through morphospace. PCA $=$ principal-component axis. 
Both our model and empirical data suggest that clines frequently trace nonlinear paths through morphospace. Arcing clines can arise when different traits change at different rates along a cline. This can occur through multiple evolutionary and genetic forces. Deterministic evolutionary processes such as selection or phenotype-dependent movement can generate unequal rates of divergence among traits, producing arcing clines. Such inequalities among traits can cause some traits to diverge more quickly along a cline and other traits more slowly. Clines therefore begin to trace a path along the first (rapidly diverging) trait axis and then shift to diverging mostly along another trait axis, resulting in an arc through morphospace. Genetic processes such as dominance and epistasis might also change the relationship between genotype and phenotype in ways that induce deviations in phenotypic-cline trajectories even when allele frequencies follow straight trajectories. Stochastic processes such as genetic drift and sampling error can also introduce variation in vector length and orientation, particularly when vectors are short. Because we do not typically expect multiple traits to experience symmetric evolutionary forces (i.e., under equal selection pressure in lake and stream or subject to equal mutation rates), we anticipate that such arcing clines are likely to be the rule rather than the exception. Furthermore, we anticipate that these findings are applicable to multiple ecological circumstances that may produce clines (e.g., variation in environment across an ecotone or the formation of a hybrid zone between alternative environments). Our simulations focused specifically on a continentarchipelago setting, but the underlying logic of our finding (that different traits diverge at different speeds across a cline) should apply to many different spatial settings.

Our reanalysis of clinal data from lake and stream stickleback confirms that trait divergence between habitats entails a shifting combination of traits as one moves across the cline. Within each of the eight lake-stream pairs we examined, successive sample sites along the stream exhibited significant differences in cumulative vector orientation and length. As a result, for all eight pairs we can reject the idea that the clines entail evolution along a single multivariatetrait axis. However, for most lake-stream pairs we can also reject the model prediction of a simple arcing cline. In many cases, the vector orientations fluctuate drastically as one moves downstream (e.g., McCreight Lake and stream). As a result, vector orientations do not tend to converge asymptotically toward a distant reference vector, as predicted by our model (fig. 4, left, vs. fig. $1 E$ ). Rather, clines tend to "wobble" through morphospace, a behavior that was not apparent in our models.

The simplest explanation for these wobbling clines might be our modest sample sizes per site. Low-power data sets confer noisy estimates of multivariate trait means along a cline, generating "wobble" in the orientation of our vector estimates. However, by using a permutation-based test of angular deviations (Collyer and Adams 2007), we were able to establish that the disparate vector orientations are significantly different $(\theta>0)$. Sampling noise may still contribute to the wobble, but is not a sufficient explanation.

A second and more likely explanation is that the "wobbly" clines we observed are an adaptive response to heterogeneous selection pressures. In our model, we made the simplifying assumption that the continent and archipelago environments each had a single optimal phenotype combination. In reality, any given habitat category (e.g., lake or stream) may itself contain subtle environmental heterogeneity (Berner et al. 2008, 2009; Izen et al. 2016). For instance, many of the streams studied here are characterized by alternating pool and riffle habitats with varying flow rates, depths, substrates, vegetation, and invertebrate communities. This within-stream environmental heterogeneity could give rise to adaptive within-stream phenotypic variation, causing the cline to fluctuate more substantially in morphospace. In support of this hypothesis, a recent survey of three lake-stream pairs found that individual fish ecomorphology (fin shape and gill raker traits) covaried with flow regime within each stream even after clinal distance from the nearest lake was controlled for (Izen et al. 2016). As a result, phenotypic variation among sites within each stream equaled or exceeded the phenotypic differences between lake and stream forms. We therefore propose that the "wobbling" clines seen in most lake-stream pairs may be attributable to environmental variation along the length of a given stream.

Fluctuating phenotypic values might also arise via phenotypic plasticity. The empirical data used here are based on wild-caught stickleback and so represent a combination of genetic and phenotypic effects. Previous common-garden studies of three lake-stream pairs (a subset of those studied here) found that lake-stream differences are, for the most part, retained in lab-reared fish and therefore are heritable (Oke et al. 2016). However, this conclusion does not exclude the possibility that environmental variation generates additional plastic changes in wild sticklebacks' traits, as seen for life-history traits in European lake-stream stickleback (Moser et al. 2015). If different areas along the stream experience different environments or have heritable differences in plasticity (genotype-environment interactions), this might plausibly generate deviations from a straight-line series of vectors in morphospace.

A fourth possibility is that wobble in cline orientation results from localized genetic drift. Drift induces (slight) random changes in mean phenotype and can therefore lead to random deviations from a major axis of lake-stream divergence. We do not here present an in-depth model of this effect, mostly because drift is not likely to generate such strong changes in vector direction in our empirical system. Esti- 
mates of effective population sizes in lake and stream stickleback suggest relatively large and recently established populations. Estimates of migration among stream sites suggest moderately high capacity for gene flow. Together, these observations should limit the role of genetic drift in generating substantial variation in phenotypes along a given cline.

A final possibility that we have not evaluated is that epistatic interactions among genes might generate nonadditive phenotypic changes along a cline. Transgressive segregation of traits occurs when multiple loci epistatically create heterozygote phenotypes that lie outside the trait range of homozygotes (deVicente and Tanksley 1993). In such cases, hybrid zones where heterozygotes are abundant might plausibly contain many phenotypically atypical individuals that fall outside the phenotypic axis defined by pure lake or stream genotypes, causing transient rotation of vectors of trait divergence.

Most likely there are additional processes that can generate wobbling or arcing multivariate clines. The goal of our model and data is not to exhaustively categorize all such processes or test particular hypotheses. Rather, our goal is to highlight the capacity for clines to proceed irregularly through multivariate space and thereby raise the questions of what processes might be responsible and what the consequences might be. To that end, we note that the number of dimensions in such an analysis is also important. To illustrate this sensitivity, we repeated our empirical analyses in bivariate space, focusing on gill raker number and relative warp 1 (arguably the most ecologically important traits). This reduced dimensionality still visually appears to yield nonlinear clines. However, for most lake-stream pairs the arcing or wobbling is not statistically significant in this twodimensional space (fig. A2; figs. A1, A2 available online). For about half of the lake-stream pairs, the vector from the lake to the first stream site points in a direction significantly different from that of all the subsequent lake-stream vectors. This suggests that admixture in the earliest sites creates unique trait combinations. In the other half of the lakestream pairs, there is no evidence for nonlinear clines in bivariate space. The lake-stream vectors apparently lengthen along a single major axis as one proceeds downstream. However, the two-dimensional morphospace for lake-stream fish might not provide sufficient power to detect angle differences between vectors by using the permutation test. Or the angles between vectors might be largely due to higher-order relative warps. The key point here is that one's inferences may depend on which traits, and how many trait dimensions, are used to study multivariate clines.

The most obvious practical consequence of arcing or irregular clines is that no single phenotypic axis can effectively document the phenotypic divergence across a landscape. The common practice of using a first principal-component axis or a discriminant-function axis to represent phenotypic change will be insufficient. The more arced (or wobbling) the cline is, the more severe this insufficiency. A second practical implication is that the curvature of multivariate clines may give some clues as to the evolutionary forces driving divergence. For instance, if arcing clines are typically caused by unequal selection among traits, then the direction of the arc may help identify traits that are especially important targets of divergent selection. However, the complex factors contributing to variation in cline shape and magnitude may often be difficult to disentangle empirically (Slatkin 1978; Barton 1999; Geroldinger and Bürger 2015).

Nonlinear multivariate clines might also play a significant role in reproductive isolation during parapatric speciation (Lande 1982). Many populations exhibit a modest degree of positive assortative mating: males and females are more likely to form pairs if they are phenotypically similar (Jiang et al. 2013). This within-population assortative mating can generate some reproductive isolation with respect to phenotypically divergent individuals from neighboring habitats. However, such isolation will usually be relatively weak, because assortative mating is typically subtle and phenotypic divergence is slight (Bolnick and Kirkpatrick 2012). However, multivariate phenotypic differences might have multiplicative effects on premating isolation between adjoining populations. If so, the speed at which multiple traits diverge along a cline (represented by our vector analysis) may have a substantial impact on the strength of reproductive isolation between adjoining populations and the location along the cline where this isolation is strongest.

In conclusion, we have shown both theoretically and empirically that clines may progress nonlinearly through phenotypic space. Given the many evolutionary and genetic processes that might cause such arcing clines, we anticipate that such curved clines will be a typical feature of spatial divergence in nature. If, on reanalysis, many other clines exhibit arcing or irregular trajectories, then the causes and consequences of such multivariate trends merit further attention.

\section{Acknowledgments}

We thank Y. Stuart for helpful comments during manuscript preparation. We thank the Texas Advanced Computing Center for computing time for simulations. We thank the anonymous reviewer, associate editor C. Caruso, and editor Y. Michalakis for their helpful comments. This work was supported by National Science Foundation grant DEB1144773.

\section{Literature Cited}

Adams, D. C., and M. L. Collyer. 2009. A general framework for the analysis of phenotypic trajectories in evolutionary studies. Evolution 65:1143-1154. 
Adrion, J. R., M. W. Hahn, and B. S. Cooper. 2015. Revisiting classic clines in Drosophila melanogaster in the age of genomics. Trends in Genetics 31:434-444.

Albert, A. Y., S. Sawaya, T. H. Vines, A. K. Knecht, C. T. Miller, B. R. Summers, S. Balabhadra, D. M. Kingsley, and D. Schluter. 2008. The genetics of adaptive shape shift in stickleback: pleiotropy and effect size. Evolution 62:76-85.

Barton, N. H. 1999. Clines in polygenic traits. Genetics Research 74:223-236.

Barton, N. H., and G. M. Hewitt. 1989. Adaptation, speciation and hybrid zones. Nature 341:497-502.

Barton, N. H., and P. D. Keightley. 2002. Understanding quantitative genetic variation. Nature Reviews Genetics 3:11-20.

Berner, D., D. C. Adams, A. C. Grandchamp, and A. P. Hendry. 2008. Natural selection drives patterns of lake-stream divergence in stickleback foraging morphology. Journal of Evolutionary Biology 21: 1653-1665.

Berner, D., A.-C. Grandchamp, and A. P. Hendry. 2009. Variable progress toward ecological speciation in parapatry: stickleback across eight lake-stream transitions. Evolution 63:1740-1753.

Berner, D., R. Kaeuffer, A.-C. Grandchamp, J. A. M. Raeymaekers, K. Räsänen, and A. P. Hendry. 2011. Quantitative genetic inheritance of morphological divergence in a lake-stream stickleback ecotype pair: implications for reproductive isolation. Journal of Evolutionarv Biology 24:1975-1983.

Berner, D., W. E. Stutz, and D. I. Bolnick. 2010. Foraging trait (co)variances in stickleback evolve deterministically and do not predict trajectories of adaptive diversification. Evolution 64:22652277.

Bolnick, D. I., and M. Kirkpatrick. 2012. The relationship between intraspecific assortative mating and reproductive isolation between divergent populations. Current Zoology 58:484-492.

Bolnick, D. I., and J. S. Paull. 2009. Morphological and dietary differences between individuals are weakly but positively correlated within a population of threespine stickleback. Evolutionary Ecology Research 11:1217-1233.

Bolnick, D. I., K. C. Shim, M. Schmerer, and C. D. Brock. 2015 Population-specific covariation between immune function and color of nesting male threespine stickleback. PLoS ONE 10(6): e0126000. doi:10.1371/journal.pone.0126000.

Bolnick, D. I., L. K. Snowberg, C. Patenia, W. E. Stutz, T. Ingram, and O. L. Lau. 2009. Phenotype-dependent native habitat preference facilitates divergence between parapatric lake and stream stickleback. Evolution 63:2004-2016.

Caisse, M., and J. Antonovics. 1978. Evolution in closely adjacent plant populations. Heredity 40:371-384.

Campitelli, B. E., and J. R. Stinchcombe. 2013. Natural selection maintains a single-locus leaf shape cline in Ivyleaf morning glory, Ipomoea hederacea. Molecular Ecology 22:552-564.

Collyer, M. L., and D. C. Adams. 2007. Analysis of two-state multivariate phenotypic change in ecological studies. Ecology 88:683-692.

- 2013. Phenotypic trajectory analysis: comparison of shape change patterns in evolution and ecology. Hystrix 24:75-83.

deVicente, M. C., and S. D. Tanksley. 1993. QTL analysis of transgressive segregation in an interspecific tomato cross. Genetics 134 585-596.

Endler, J. A. 1977. Geographic variation, speciation, and clines. Princeton University Press, Princeton, NJ.

Fitzpatrick, B. M. 2013. Alternative forms for genomic clines. Ecology and Evolution 3:1951-1966.
Geroldinger, L., and R. Bürger. 2015. Clines in quantitative traits: the role of migration patterns and selection scenarios. Theoretical Population Biology 99:43-66.

Gilchrist, G. W., R. Huey, and L. Serra. 2001. Rapid evolution of wing size clines in Drosophila subobscura. Genetica 112-113:273-286.

Gompert, Z., and C. A. Buerkle. 2011. Bayesian estimation of genomic clines. Molecular Ecology 20:2111-2127.

Grahame, J. W., C. S. Wilding, and R. K. Butlin. 2006. Adaptation to a steep environmental gradient and an associated barrier to gene exchange in Littorina saxatilis. Evolution 60:268-278.

Hanson, D., J. S. Moore, E. Taylor, R. Barrett, and A. Hendry. 2016. Assessing reproductive isolation using a contact zone between parapatric lake-stream stickleback ecotypes. Journal of Evolutionary Biology.

Hendry, A. P., and E. B. Taylor. 2004. How much of the variation in adaptive divergence can be explained by gene flow? an evaluation using lake-stream stickleback pairs. Evolution 58:2319-2331.

Hendry, A. P., E. B. Taylor, and J. D. McPhail. 2002. Adaptive divergence and the balance between selection and gene flow: lake and stream stickleback in the Misty system. Evolution 56:11991216.

Hoffmann, A. A., A. Anderson, and R. Hallas. 2002. Opposing clines for high and low temperature resistance in Drosophila melanogaster. Ecology Letters 5:614-618.

Izen, R., Y. E. Stuart, Y. Jiang, and D. I. Bolnick. 2016. Coarse-and fine-grained phenotypic divergence among threespine stickleback from alternating lake and stream habitats. Evolutionary Ecology Research 17:437-457.

Jiang, Y., D. I. Bolnick, and M. Kirkpatrick. 2013. Widespread positive assortative mating within animal populations. American Naturalist 181:E125-E138.

Jiang, Y., L. Torrance, C. L. Peichel, and D. I. Bolnick. 2015. Divergent rheotaxis contributes to divergent habitat preferences between lake and stream threespine stickleback. Evolution 69:2517-2524.

Kaeuffer, R., D. Bolnick, A. P. Hendry, and C. L. Peichel. 2012. Convergence and non-convergence in ecological, phenotypic, and genetic divergence across replicate population pairs of lake and stream stickleback. Evolution 66:402-418.

Lande, R. 1982. Rapid origin of sexual isolation and character divergence in a cline. Evolution 36:213-223.

Lavin, P. A., and J. D. McPhail. 1986. Adaptive divergence of trophic phenotype among freshwater populations of the threespine stickleback (Gasterosteus aculeatus). Canadian Journal of Fisheries and Aquatic Sciences 43:2455-2463.

1993. Parapatric lake and stream sticklebacks on northern Vancouver Island: disjunct distribution or parallel evolution? Canadian Journal of Zoology 71:11-17.

Lenormand, T. 2002. Gene flow and the limits to natural selection. Trends in Ecology and Evolution 17:183-189.

Lohman, B. K., D. Berner, and D. I. Bolnick. 2017. Data from: Clines arc through multivariate morphospace. American Naturalist, Dryad Digital Repository, http://dx.doi.org/10.5061/dryad.25h9d.

Moore, J.-S., J. L. Gow, E. B. Taylor, and A. P. Hendry. 2007. Quantifying the constraining influence of gene flow on adaptive divergence in the lake-stream threespine stickleback system. Evolution 61:2015-2026.

Moore, J.-S., and A. P. Hendry. 2005. Both selection and gene flow are necessary to explain adaptive divergence: evidence from clinal variation in stream stickleback. Evolutionary Ecology Research 7:871-886 
Moser, D., B. Kueng, and D. Berner. 2015. Lake-stream divergence in stickleback life history: a plastic response to trophic niche differentiation? Evolutionary Biology 42:328-338.

Oke, K., M. Bukhari, R. Kaueffer, G. Rolshausen, K. Räsänen, D. I. Bolnick, C. L. Peichel, and A. P. Hendry. 2016. Does plasticity enhance or dampen phenotypic parallelism? a test with three lakestream stickleback pairs. Iournal of Evolutionary Biology 29:126143.

Peichel, C. L., K. S. Nereng, K. A. Ohgi, B. L. E. Cole, P. F. Colosimo, C. A. Buerkle, D. Schluter, and D. M. Kingsley. 2001. The genetic architecture of divergence between threespine stickleback species. Nature 414:901-905.

Reimchen, T. E., E. M. Stinson, and J. S. Nelson. 1985. Multivariate differentiation of parapatric and allopatric populations of threespine stickleback in the Sangan River watershed, Queen Charlotte Islands. Canadian Journal of Zoology 63:2944-2951.

Sharpe, D. M. T., K. Räsänen, D. Berner, and A. P. Hendry. 2008. Genetic and environmental contributions to the morphology of lake and stream stickleback: implications for gene flow and reproductive isolation. Evolutionary Ecology Research 10:849-866.

Slatkin, M. 1978. Spatial patterns in the distributions of polygenic characters. Journal of Theoretical Biology 70:2013-2028.
1985. Gene flow in natural populations. Annual Review of Ecology and Systematics 16:393-430.

Stinchcombe, J. R., C. Weinig, M. Ungerer, K. M. Olsen, C. Mays, S. S. Halldorsdottir, M. D. Purugganan, and J. Schmitt. 2004. A latitudinal cline in flowering time in Arabidopsis thaliana modulated by the flowering time gene FRIGIDA. Proceedings of the National Academv of Sciences of the USA 101:4712-4717.

Stock, A. J., B. E. Campitelli, and J. R. Stinchcombe. 2014. Quantitative genetic variance and multivariate clines in the Ivyleaf morning glory, Ipomea hederacea. Philosophical Transactions of the Roval Society B 369:20130259. doi:10.1098/rstb.2013.0259.

Thompson, C. E., E. B. Taylor, and J. D. McPhail. 1997. Parallel evolution of lake-stream pairs of threespine sticklebacks (Gasterosteus) inferred from mitochondrial DNA variation. Evolution 51:1955-1965.

Umina, P. A., A. R. Weeks, M. R. Kearney, S. W. McKechnie, and A. A. Hoffmann. 2005. A rapid shift in a classic clinal pattern in Drosophila reflecting climate change. Science 308:691-693.

Associate Editor: Christina M. Caruso Editor: Yannis Michalakis

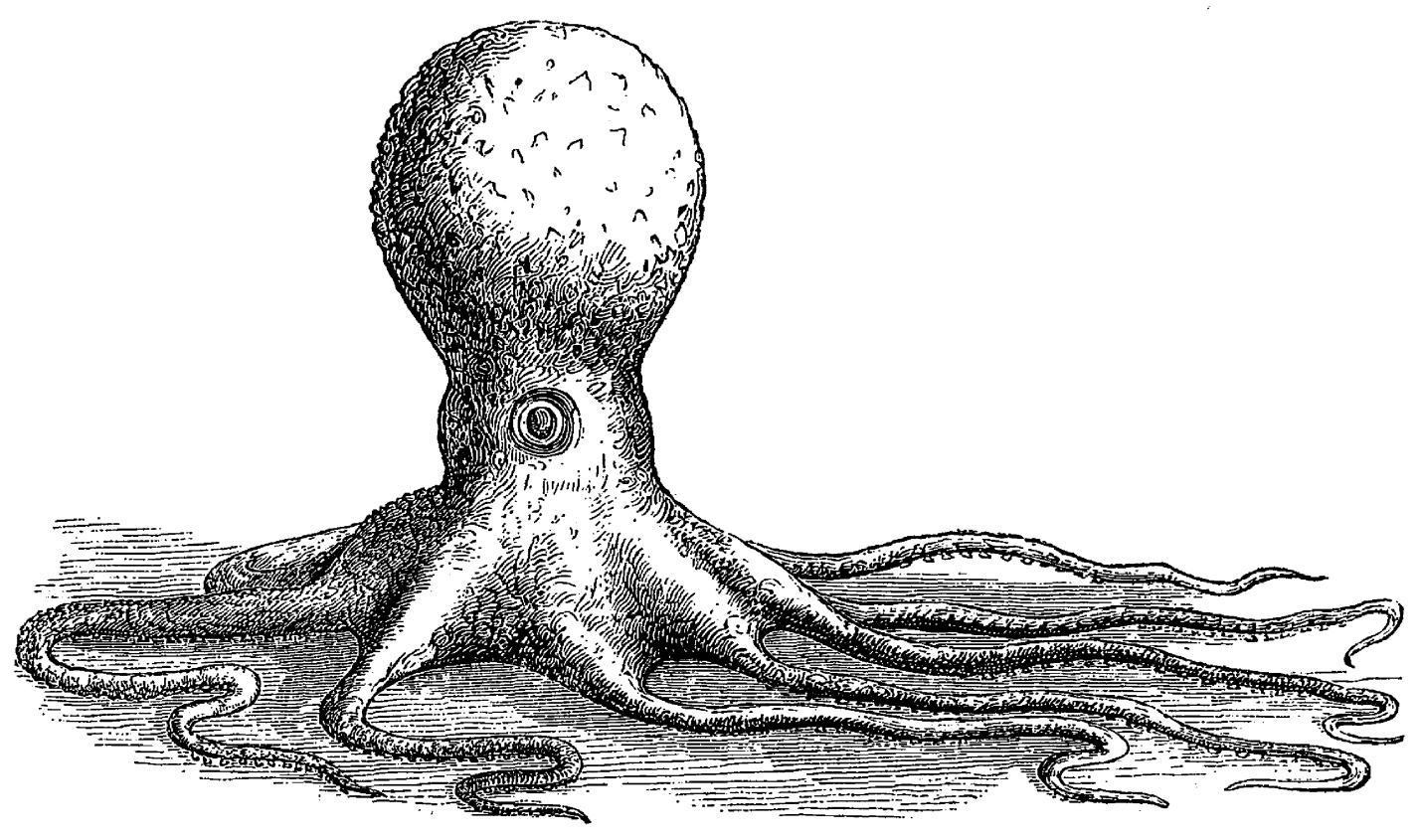

"It was during my first visit to Brazil, that one day, while busily engaged in examining a little town on the coast called Guarapary, my eye fell on an object in a shallow tide-pool, packed away in the crevice of the reef, which excited my curiosity. I could see nothing but a pair of very bright eyes; but, concluding that the eyes had an owner, I determined very rashly to secure him. . . . I put my hand down very quietly so as not to ruffle the water, when, suddenly, to my surprise, it was seized with a pressure far too ardent to be agreeable, and I was held fast. I tugged hard to get away, but this uncivil individual, whoever he was, evidently had as strong a hold on the rocks as he had on my hand, was not easily to be persuaded to let go of either. At last, however, he became convinced that he must choose between us, and so let go his hold upon the rocks, and I found clinging to my right hand, by his long arms, a large octopod cuttle-fish, resembling the one figured." From "A Chapter on Cuttle-Fishes" by Lucie L. Hartt (The American Naturalist, 1869, 3:257-261). 